\title{
LIST OF REFEREES
}

We thank those who have given their time to referee for Diabetologia, all of whom are listed on our website (http://www. diabetologia-journal.org/referees.html). Special thanks are due to our key referees, listed below, who have each provided at least three timely and well-rated reports over the past year.

\author{
Al-Hasani H; Nuthetal \\ Allen T; Melbourne \\ Andrikopoulos S; Heidelberg Heights \\ Atkinson M; Gainesville \\ Avogaro A; Padua \\ Badenhoop K; Frankfurt \\ Bailey $\mathrm{C}$; Birmingham \\ Balkau B; Villejuif \\ Baltrusch S; Rostock \\ Barres R; Sydney \\ Barrett E; Charlottesville \\ Bernal-Mizrachi E; Michigan \\ Bhatnagar D; Oldham \\ Biden T; Sydney \\ Birnbaum M; Philadelphia \\ Blüher M; Leipzig \\ Bonadonna R; Verona \\ Bonifacio E; Dresden \\ Bonner-Weir S; Boston \\ Bonnet F; Rennes \\ Bouwens L; Brussels \\ Braun M; Oxford \\ Broadbent E; Auckland \\ Brubaker P; Toronto \\ Buchanan T; Los Angeles \\ Busik J; East Lansing \\ Byrne C; Southampton \\ Calcutt N; La Jolla \\ Candido R; Trieste \\ Carlsson P-O; Uppsala \\ Carstensen B; Gentofte \\ Cauchi S; Lille \\ Chaturvedi N; London \\ Colman P; Parkville \\ Cooper G; Auckland \\ Cooper M; Melbourne \\ Coughlan M; Melbourne \\ Currie C; Cardiff \\ Springer
}

Dahlquist G; Umeå

Davis T; Fremantle

Dekker J; Amsterdam

Del Prato S; Pisa

Dotta F; Siena

Drucker D; Toronto

Duvillie B; Paris

Eckel J; Düsseldorf

Eisenbarth G; Aurora

Eliasson L; Malmö

Elsner M; Hanover

Eriksson J; Helsinki

Ewings P; Taunton

Fadini G; Padua

Febbraio M; Melbourne

Ferrannini E; Pisa

Ferreira I; Maastricht

Ferrer J; Barcelona

Feskens E; Wageningen

Finucane F; Cambridge

Flanagan S; Exeter

Flatt P; Coleraine

Fleming T; Heidelberg

Folli F; San Antonio

Forbes J; Melbourne

Formisano P; Naples

Franks P; Malmö

Freathy R; Exeter

Fritsche A; Tübingen

Froguel P; Lille

Fürnsinn C; Vienna

Game F; Nottingham

Gardiner N; Manchester

Gastaldelli A; Pisa

Gerstein H; Hamilton

Giaccari A; Rome

Gill J; Glasgow
Giunti S; Turin

Glund S; Biberach an der Riss

Grant M; Gainesville

Grant S; Philadelphia

Grarup N; Gentofte

Grauslund J; Odense

Gribble F; Cambridge

Guigas B; Leiden

Haffner S; San Antonio

Hakonarson H; Philadelphia

Haller H; Hanover

Hamilton-Shield J; Bristol

Hammarstedt A; Gothenburg

Hammes H; Mannheim

Hanley A; Toronto

Harjutsalo V; Helsinki

Harris P; New York

Hawley J; Melbourne

Heise T; Neuss

Heller S; Sheffield

Hemkens L; Cologne

Henry B; San Diego

Hivert M-F; Boston

Højlund K; Odense

Holst J; Copenhagen

Home P; Newcastle upon Tyne

Homo-Delarche F; Paris

Hribal M; Catanzaro

Hull R; Seattle

Humpert P; Heidelberg

Hutton J; Aurora

In't Veld P; Brussels

Iversen M; Bergen

Jenkins A; Melbourne

Jerums G; Heidelberg

Jiang Q; Stockholm

Johnson J; Vancouver 
Johnson J; Edmonton

Joost H; Bergholz-Rehbrücke

Jowett J; Melbourne

Kaestner K; Philadelphia

Kahn R; Alexandria

Kahn S; Seattle

Kantartzis K; Tübingen

Kay T; Fitzroy

Kelly D; Fitzroy

Kerr P; Melbourne

Kirwan J; Cleveland

Kitamura T; Gunma

Knip M; Helsinki

Langenberg C; Cambridge

Lenzen S; Hanover

LeRoith D; New York

Levidiotis V; Camperdown

Lindsay R; Glasgow

Ling C; Malmö

Livingstone S; Dundee

Loos R; Cambridge

Ludvik B; Vienna

Lyssenko V; Malmö

Lystig T; Ridgefield

MacIsaac R; West Heidelberg

Madeddu P; Bristol

Madsbad S; Hvidovre

Maechler P; Geneva

Maedler K; Bremen

Mahon J; London

Malecki M; Krakow

Mandrup-Poulsen T; Gentofte

Manrique C; Columbia

Marchetti P; Pisa

Mari A; Padua

Marshall S; Newcastle upon Tyne

Mayer D; Heidelberg

McKnight A-J; Belfast

Meier J; Bochum

Menne J; Hanover

Meyer G; Witten

Miele C; Naples

Mingrone G; Rome

Monami M; Florence

Monnier L; Montpellier

Mulder H; Malmö

Näslund E; Stockholm

Nathan D; Boston
Nauck M; Bad Lauterberg

Nawroth P; Heidelberg

Nelson R; Phoenix

Neufer D; Greenville

Newsholme P; Dublin

Ng M; Shatin

Nissen S; Cleveland

Noble J; Oakland

Orchard T; Pittsburgh

Ouwens M; Düsseldorf

Owen K; Oxford

Ozanne S; Cambridge

Pacini G; Padua

Patti M-E; Boston

Peakman M; London

Perseghin G; Milan

Petrie J; Glasgow

Pettitt D; Santa Barbara

Pihlajamäki J; Boston

Pollin T; Baltimore

Polychronakos C; Montreal

Porta M; Turin

Pouwer F; Amsterdam

Preiss D; Glasgow

Prentki M; Montreal

Ramachandran A; Chennai

Randeva H; Coventry

Rasmussen L; Aarhus

Rathmann W; Düsseldorf

Reimann F; Cambridge

Renehan A; Manchester

Renström E; Malmö

Ristow M; Bergholz-Rehbrücke

Robertson P; Seattle

Roden M; Düsseldorf

Roep B; Leiden

Rossing P; Gentofte

Rustenbeck I; Braunschweig

Rutter G; London

Sandow J; Glashütten

Savage D; Cambridge

Schalkwijk C; Maastricht

Schloot N; Düsseldorf

Schrauwen P; Maastricht

Schuit F; Leuven

Schulze M; Freising

Schürmann A; Nuthetal

Scott F; Ottawa
Semple R; Cambridge

Senneville E; Tourcoing

Service F; Rochester

Sesti G; Catanzaro

Sharp S; Cambridge

Sorenson R; Minneapolis

Staiger H; Tübingen

Stehouwer C; Maastricht

Steinberg G; Hamilton

Stephens J; Baton Rouge

Stitt A; Belfast

Stolk R; Groningen

Stratton I; Cheltenham

't Hart L; Leiden

Tanti J-F; Nice

Taylor R; Newcastle upon Tyne

Tesch G; Clayton

Thorens B; Lausanne

Thornalley P; Coventry

Tilg H; Innsbruck

Timpson N; Bristol

Todd J; Cambridge

Tripathy D; San Antonio

Ulianich L; Naples

Ullrich S; Tübingen

Ungaro P; Naples

Uusitupa M; Kuopio

Vaarala O; Linköping

Verchere B; Vancouver

Vidal H; Lyons

Voight B; Cambridge

von Herrath M; La Jolla

Walker M; Newcastle upon Tyne

Wang M; Thousand Oaks

Warren R; Edinburgh

Watt M; Clayton

Weedon M; Exeter

Weigert C; Tübingen

Weir G; Boston

Welsh N; Uppsala

Werstuck G; Hamilton

Wherrett D; Toronto

Witte D; Gentofte

Wojtaszewski J; Copenhagen

Wong S; Bristol

Zethelius B; Uppsala

Zeyda M; Vienna 